\title{
Multimodal fatigue progress monitoring of construction steel elements
}

\author{
by B. Szymanik, G. Psuj, P. Lopato, M. Maciusowicz, M. Herbko
}

\author{
Department of Electrical and Computer Engineering, Faculty of Electrical Engineering, West Pomeranian \\ University of Technology, al. Piastów 17, 70-310 Szczecin, Poland, szymanik@zut.edu.pl
}

\begin{abstract}
In this paper a new approach to state assessment of steel structures under a dynamic load conditions is presented. In order to monitor different aspects of material's structural changes during fatigue process, two sets of measuring methods allowing both global and local evaluation of the structure are utilized. In order to monitor general changes state under cyclic loading conditions, together with stress level and formation of plastic deformation areas, an infrared thermography (IRT) and low frequency range electromagnetic methods related with monitoring of AC magnetization process (EM) are used. Then for examination of critical plastic deformation area under static loading conditions a IRT method with support of microwave (MW) one are applied. The combination of the three methods of NDT (Non-Destructive Testing) techniques allows to obtain the full description of the tested structure.
\end{abstract}

\section{Introduction}

Continuous development of industry and new technologies forces the production of increasingly complex and specialized steel structures, which are the core of manufactured buildings and machinery. Such structures are subjected to large and mainly fluctuating mechanical stresses which in consequences lead to certain failure. This process of material fatigue degradation is one of the main causes of mechanical damages occurring nowadays in industry. Such damages can cause big financial losses and even be the cause of the disasters demonstrating serious consequences. Therefore the proper assessment of fatigue process course and prediction of future operating time of the construction is one of the main problems of maintenance services. To minimize the risk of unexpected failures, more and more often, the steel construction elements are subjected to continuous monitoring and evaluation of theirs structure's state using different methods of NDT.

One can distinguish three major phases of material's degradation which relate to microstructural change ending with a fatigue breakthrough stage, then development of crack growth area in the material and finally to destruction of the material [1]. However the course of the fatigue process (dynamics of each stage progress) is depended on many various factors related to the structure of the material such as the state of the microstructure, homogeneity of the material, the smoothness of the surface, residual stress condition and the level of loading forces [2]. Therefore, during the selection of nondestructive testing methods contributing to the process of assessing the degradation state of the material several factors must be taken into account. It is important to analyze what type of defects may occur in the examined construction together with the capability of the NDT technique to distinguish between the areas having the flaws and without them. Furthermore, the possibility of carrying out a rapid assessment of the overall condition of the structure as well as obtaining detail information for the selected critical areas has to be also considered. In addition, construction elements are subjected to varying conditions as a consequence of constant and alternating loads occur during fatigue life. In fact, during evaluation of the material degradation both loading factors should be taken into account. Therefore in this paper the evaluation of influence of both types of forces will be carried out. According to the above requirements the concept of combining the capabilities of three different measuring methods for local and global evaluation of steel structure degradation under static and dynamic loading conditions respectively during fatigue life will be presented. Recently the thermographic global state observation of fatigue changes along with the electromagnetic technique were carried out [3]. The achieved results confirmed the possibility of utilization of both methods for general evaluation of fatigue progress, however there was some drop of sensitivity in local state assessment. Therefore in this paper to observe the global state of construction and monitoring of stress concentration and plastics deformation areas leading to selection the critical areas, the IRT method together with EM methods (AC magnetization and the Barkhausen noise methods) will be used. Furthermore, in the critical areas where it will be important to determine precisely the range of microcracks growth the IRT with support of the MW technique will be applied.

\section{Experimental set-up}

In order to estimate the impact of each load type on degradation progress the experiment were carried out separately under static and dynamic conditions. First the observation of material degradation under of static and then dynamic cyclic loading conditions will be presented and discussed. 


\subsection{1/qirt.2016.039}

During the experiments two types of construction steel were utilized: St3s - in accordance to Polish standards PN-88 H-84020 (an equivalent for S235JRG2 in standards EN 10025) and S355J2G3 - in accordance to standards EN10025. The views of the samples shapes with depicted dimensions and region of interests were presented in Fig. 1. In case of the static loading experiments, in order to concentrate the structure's changes and precisely monitor locally the area of the greatest deformations two smooth triangle notches were produced on both sides of the samples (Fig 1a). In case of the dynamic tests the utilized shape of the sample allowed to achieve more uniform distributions of the material changes on greater area, what help to observe globally more precisely small changes in material structure.

In both cases in order to observe the general changes of material's structure, the Infrared thermography inspection was carried out. Additionally for monitoring deformations under static loading conditions microwave technique while in case of dynamic loading conditions low frequency electromagnetic one were applied.

The first of proposed methods is the active IRT (in the first experiment - static loading monitoring - the external energy source is mechanical stress. In the second experiment - damage estimation caused by cyclic loading conditions the halogen lamp excitation was used). It has been already shown, that this technique may be successfully used in the steel structures analysis (defects, especially micro cracks detection, welding and other metallurgical processes control, etc.) [4 - 6]. The mechanism of thermal contrast production in the steel structures under load (in this case the static load) can be described as follows: the heat is generated due to internal friction causing the energy dissipation as a result of tension and compression, which can be described by the mechanical hysteresis. Internal structure of the tested material, in particular defects (such as cracks or weakening of the structure caused by the mechanical stress) affects the heat generation. In case of dynamic load the active thermography with halogen lamp will be used to examine the sample. The material's thermal properties are affected during the loading conditions, by steel microstructure damages. Monitoring of the heating and cooling process allows for global assessment of material state. Sensitive infrared camera is used to observe the temperature distribution on the sample's surface. The high temperature resolution allows to observe even small internal inhomogeneities in the form of the overheated or colder places, showing the location, size and type of defects.

The IRT method was supported by microwave technique during the monitoring of the sample's static loading process. In this method the microwave patch sensors is used to monitoring the stress and crack growth in conductive material $[8,9]$. In case of stress monitoring microstrip sensor has its own ground plane (it is a part of the sensor). In case of surface state and crack growth monitoring evaluated conductive material acts as a ground plane of patch antenna sensor. We proposed a microstrip structure which is applicable in both cases. Such structure should be fixed in places, where probability of crack existence is high, eg. in vicinity of holes and slots. The presence of a crack in the examined material as well, as elongation of the patch caused by stress or crack opening, influences its electric length. Resulting resonant frequency shift can be monitored by microwave vector network analyzer (VNA). The frequency shift is linearly related to applied stress and crack length $[9,10]$.

In case of cyclic loading the active thermography with halogen lamps excitation was completed by electromagnetic testing techniques. Due to the electromagnetic properties of steels utilized for the constructions, the use of magnetic testing methods becomes a natural solution for evaluation of theirs damage stage. Material properties are influenced by the stress and fatigue loading conditions. It is known that microstructure features affect the magnetic one [1]. Therefore, by monitoring changes of magnetic properties, the damage state of the material can be evaluated. Various magnetic properties can be considered in the process of damage state evaluation [1, 7]. Hysteresis loop parameters such as remanence or coercivity as well as Barkhausen noise characteristic values such as signal's energy shows good correlation with the damage state of the material.

a)

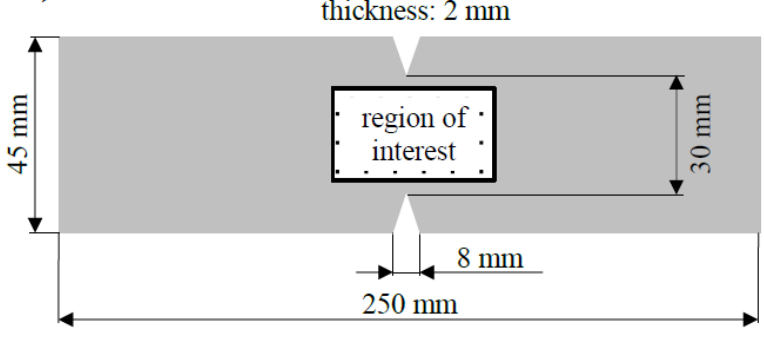

b)

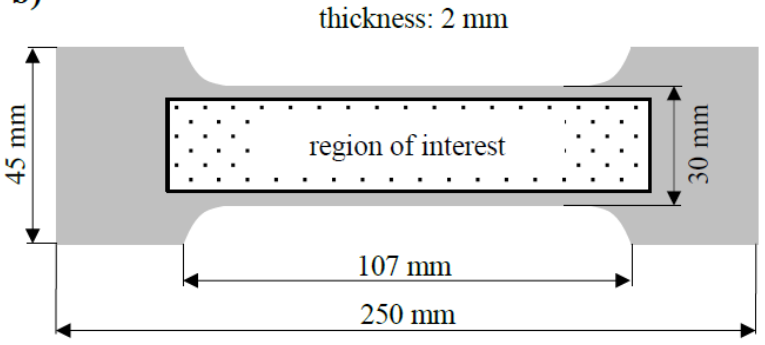

Fig. 1. View of the samples with depicted dimensions utilized in the experiments made of: a) St3s steel, b) S355J2G3. 

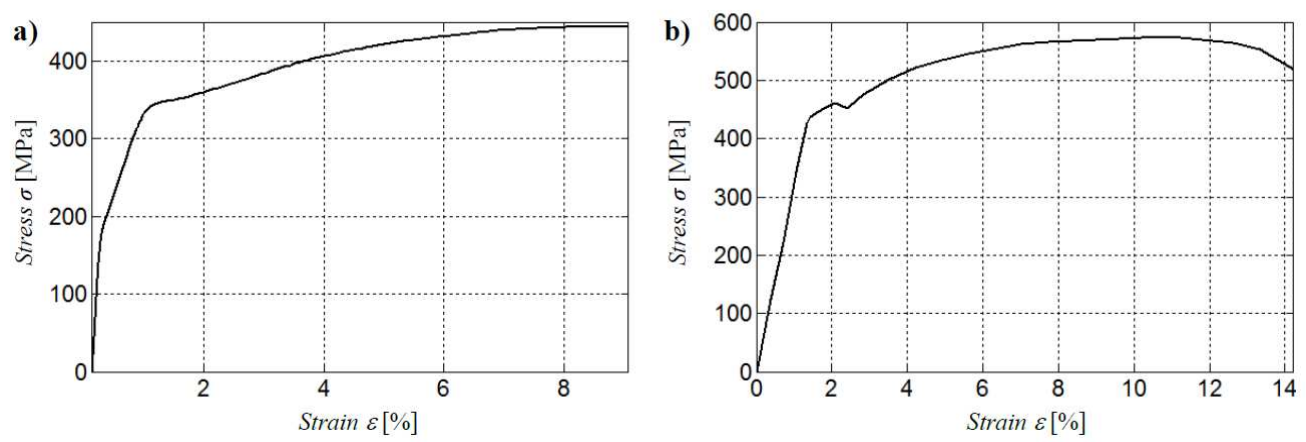

Fig. 2. Stress-strain curve obtained during material examination for: a) St3s steel and b) S355J2G3 steel.

\subsection{Static loading experiment}

The experiment was carried out for st3s steel samples (Fig. 1a). The sample was fixed in the stress machine and the material's structure changes were observed while carrying out the loading process (in the longitudinal direction). The stress-strain curve of the st3s steel obtained during the laboratory tests is presented in Fig. 2. The steel's yield limit is around $235 \mathrm{MPa}$. During the experiment two NDT methods were used: IRT and MW. The measuring system is shown in Fig. 3. IR camera is positioned to observe the loaded sample with attached microstrip antenna sensor. The microwave vector network analyzer (VNA) is used to measure the reflection coefficient $S_{11}$.

IR technique can be recognized as very effective in case of monitoring the static loading process. Well known elastic and plastic heat effects (thermomechanical coupling) accompany the observed process and the temperature changes can be observed using thermovision camera. Generally the temperature changes can be positive, negative or equal zero according to the kind of deformation (compression, elongation, shear). In this part of the experiment the sample is subjected to the static loading. During this process the plastic deformation occurs causing the generation, motion and annihilation of material's crystal lattice defects, which is always connected with energy dissipation observed as the temperature rise.

In case of MW technique, an applied stress changes the dimensions of the microstrip patch sensor fixed on materials surface, resulting in the change of its electrical length and frequency domain parameters like resonant frequency $f_{r}$ [8]-[12]. Structure and dimensions of proposed sensor are presented in Fig. 4. It is based on a standard rectangular patch antenna commonly utilized in wireless communication [13]. We modified this structure by cutting off rectangular area in ground plane under the patch (Fig. 4 right side). This enabled us to observe both strain and changes in materials surface caused by external stress (eg. surface porosity growth or crack formation and growth).

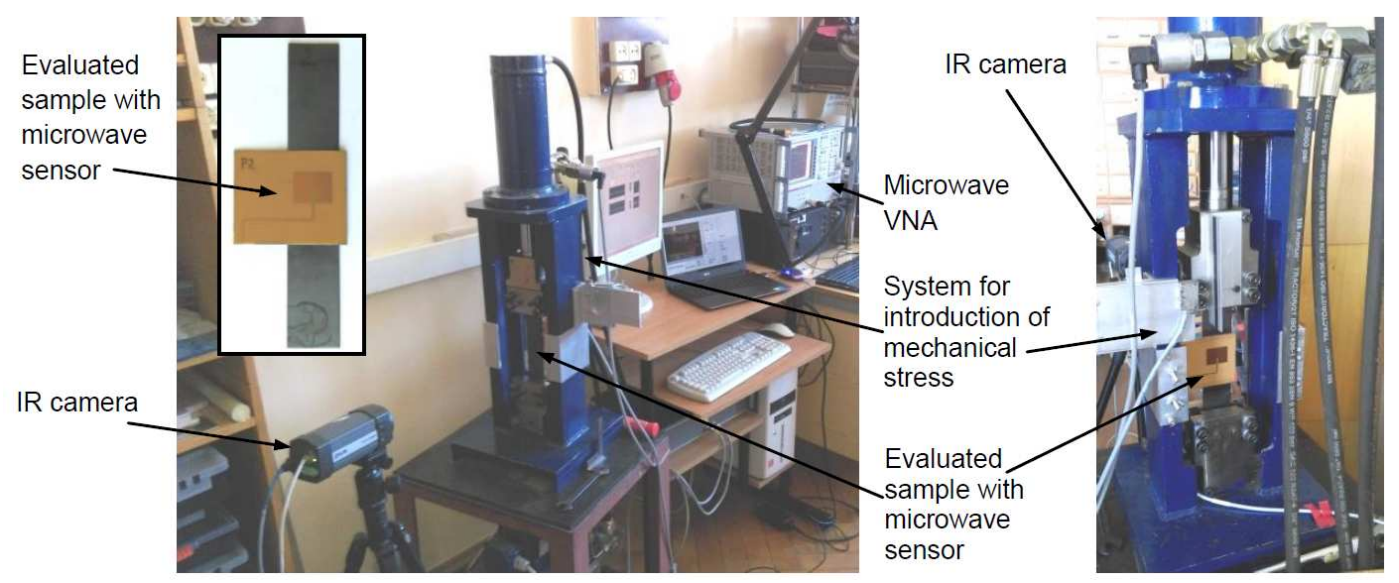

Fig. 3. Photo of measuring system for evaluation of the fatigue produced under static loading conditions. 


\subsection{1/qirt.2016.039}

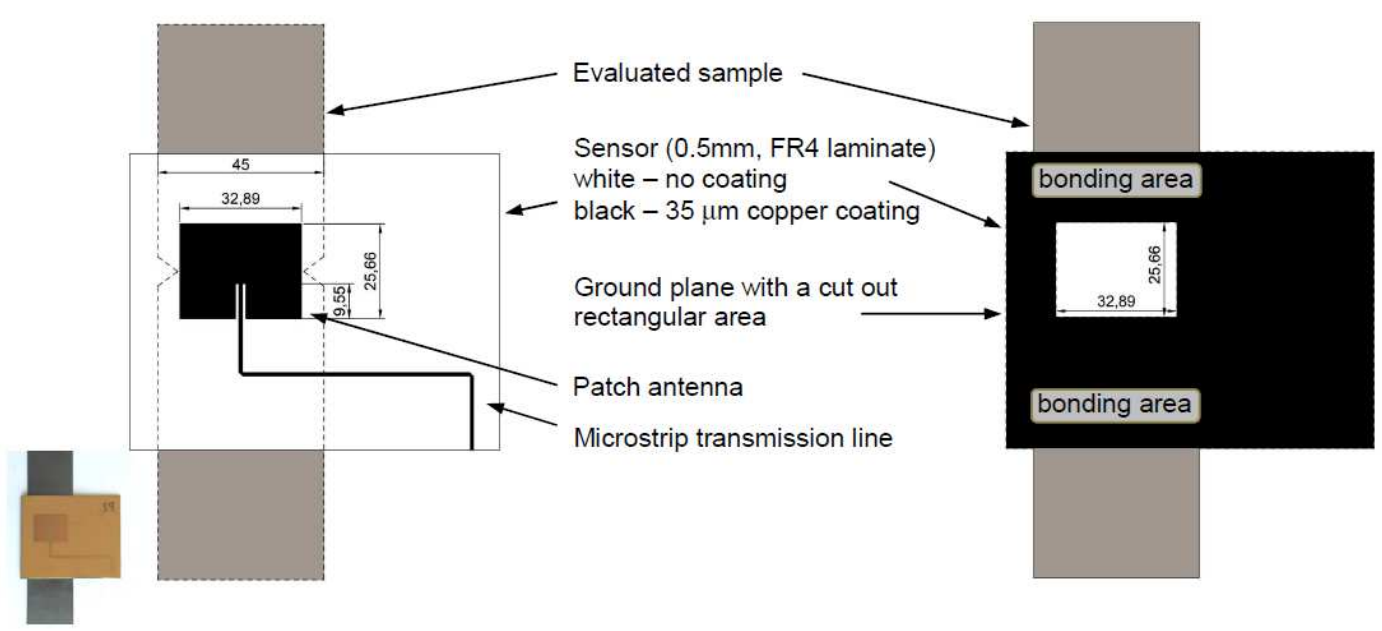

Fig. 4. Microwave microstrip sensor fixed on the evaluated steel sample: front side view (left) and back side view (right). Dimensions are presented in [mm].

\subsection{Cyclic loading experiment}

The experiment was carried out for a planar specimen made of structural steel S355J2G3. The minimum yield strength of this material was $355 \mathrm{MPa}$. The sample was tensile deformed in a longitudinal direction using cyclic loading machine with a maximum loading strength around $300 \mathrm{MPa}$. At the beginning the specimen was annealed in air at $300^{\circ} \mathrm{C}$ for one hour and then air cooled. The total number of $5,4 \cdot 10^{5}$ fatigue cycles were applied before the final evaluation of the sample was made. To evaluate fatigue damages IRT together with the BN observation and AC magnetization methods (EM methods) were used.

In order to evaluate the fatigue damages using IR technique, the experimental setup shown in Fig. 5 was used. The halogen lamp was used to heat the sample in the transition mode. The sample was coated with black paint, to remove the reflection effects observed for shiny surfaces and color inhomogeneities, causing the observation errors due to different emissivity factors. and fixed into the frame protecting the camera from the direct halogen radiation. The heating pulse duration was fixed for 15 second. After that, the sample was observed for 60 seconds more. 60 images per second were acquired, which is maximum amount for this camera. As a result the sequences of thermograms for heating phase and cooling phase were obtained.

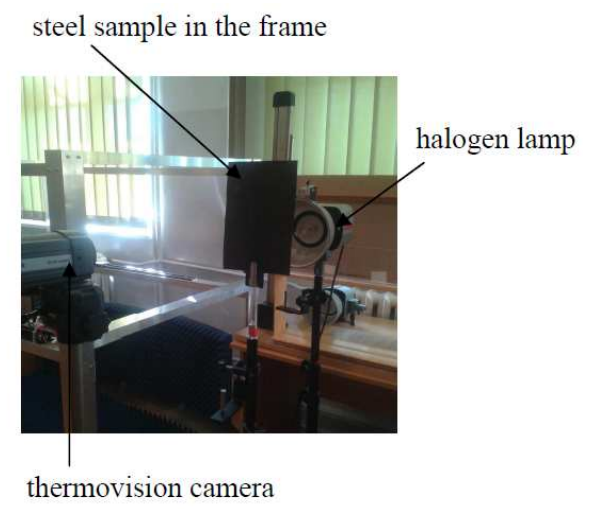

Fig. 5 Photo of the IRT system configuration

The view of experiment setup and block diagram of the EM system is presented in Fig. 6. The EM system consists of a multi-sensor transducer. The transducer is made of excitation and pick-up sections. The magnetizing field is generated by the excitation coil wound on C-shaped ferrite core. The pick-up section set-up allows to observe multiple parameters set derived from ACM and BN signals simultaneously. The signals related to magnetic field and magnetic induction were sensed respectively by a Hall effect sensor placed between the pole pieces and a pick-up B-coil wound on one column of the core. The Barkhausen noise signal is received by two coils wound on rod ferrite core connected in differential mode placed in middle of transducer. The first coil is attached to the material so it measures useful signal, 
while the second one is placed in some distance from it. This configuration allows to minimize the influence of unwanted external distortions on measured signals. During the experiments the examined sample was magnetized with the frequency of $30 \mathrm{~Hz}$. The measurements were carried out in two axis over the area marked as region of interest in Fig. $1 \mathrm{~b}$.

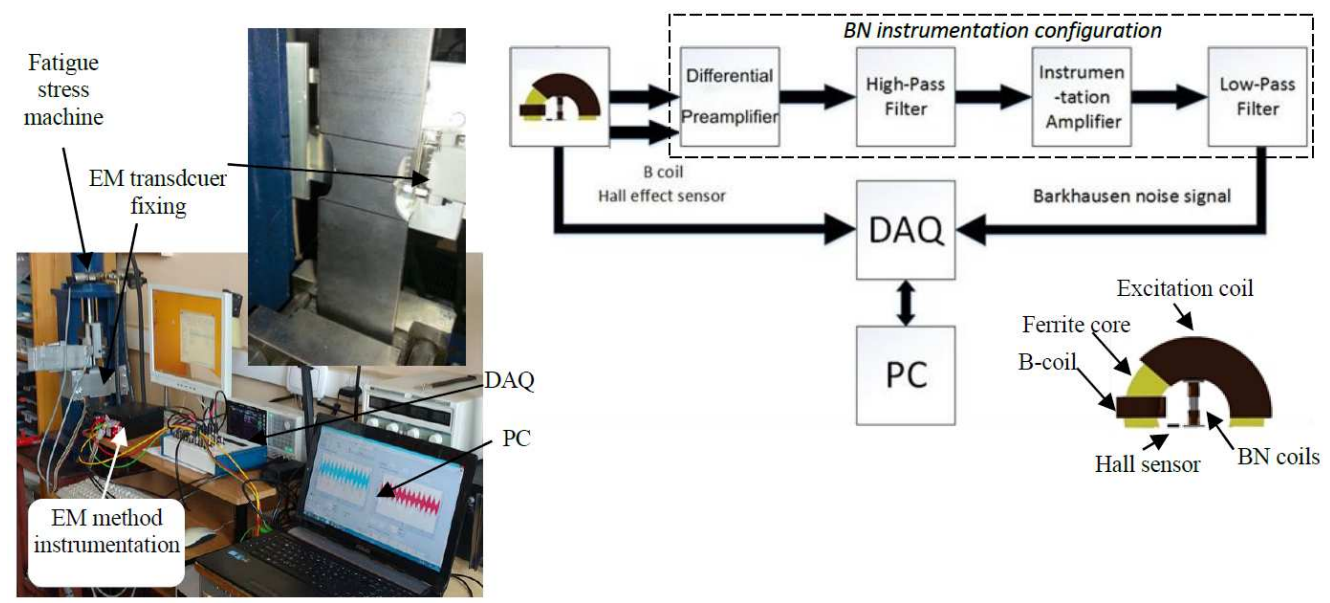

Fig. 6. Photo and block diagram of the EM system configuration

\section{Experimental results}

\subsection{Deformation evaluation caused by static load}

To monitor the temperature changes within the sample during the static loading process, FLIR 325A thermovision camera was used. The sequences of images were recorded during the set stress value change. As a result the sets of sequences for every stress step were obtained, ready for further processing. In each image the chosen region of interest (as shown if Fig. 7a) was cut and analyzed. Several parameters can be computed for each cut image data. Fig. 7b presents the standard deviation value vs. the stress value (for each image in the sequence recorded for every stress value the standard deviation was computed, then the mean value was taken to obtain the measured point). The data was normalized. It can be noticed, that the elastic and plastic region are easily distinguishable, as compared to strainstress curves presented in Fig. 2. After the steel's yield point (235MPa), the increment of the standard deviation is visible. The rapid growth of the value is seen after $350 \mathrm{MPa}$. To make the plastic region observation more detailed the set stress step was reduced.

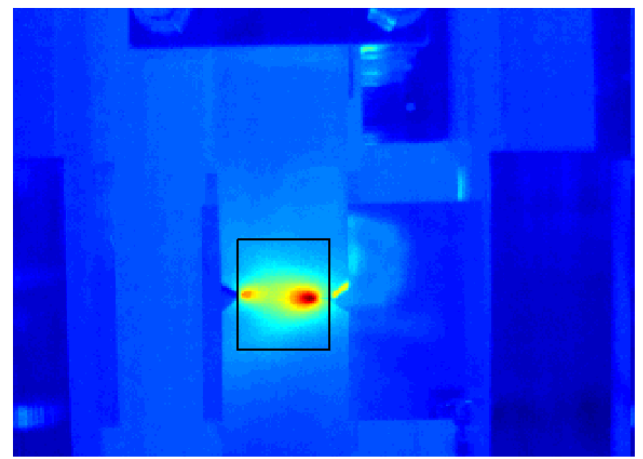

a)

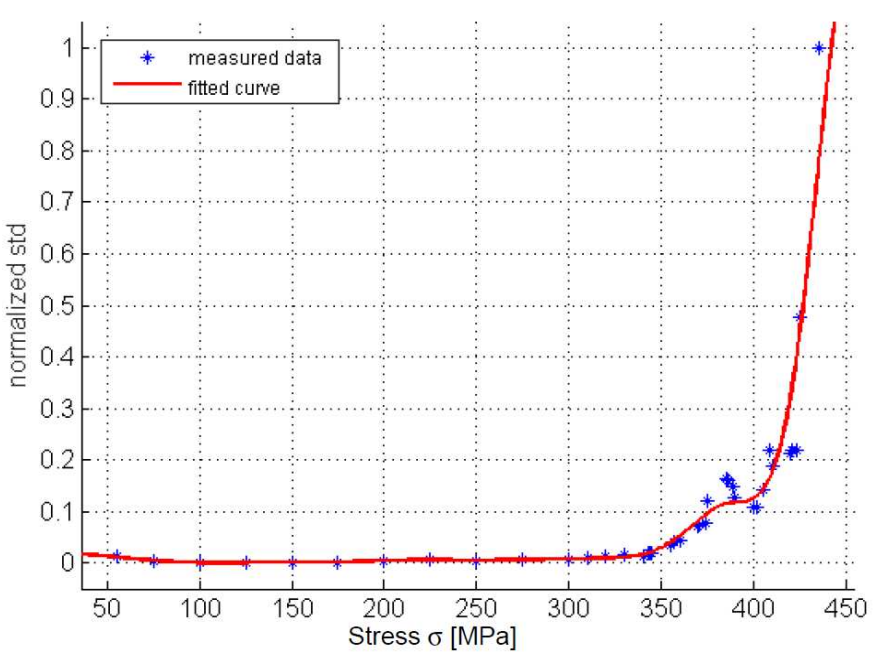

b)

Fig. 7 a) the ROI for inspected sample, b) normalized standard deviation versus stress plot 
Simultaneously the microstrip antenna was fixed to the sample, thus the secondary measurements could be done in the same time. In this two sensor approach, the possibility of similar results in case of elastic and plastic regions distinguishing was interesting.

a)

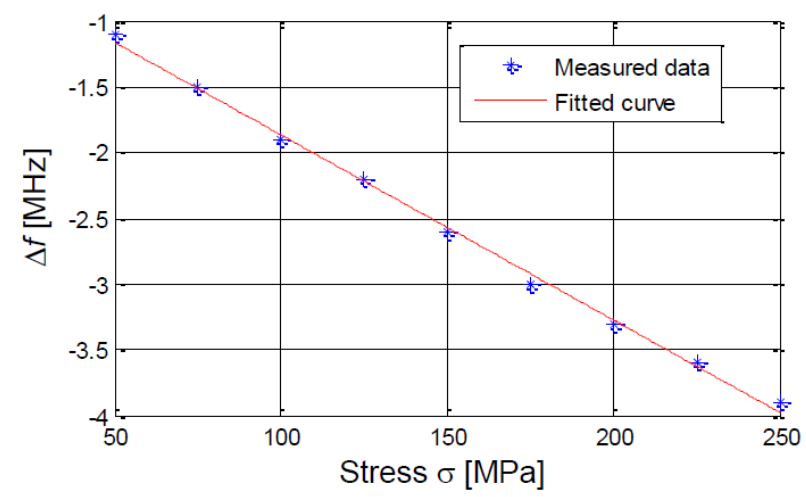

b)

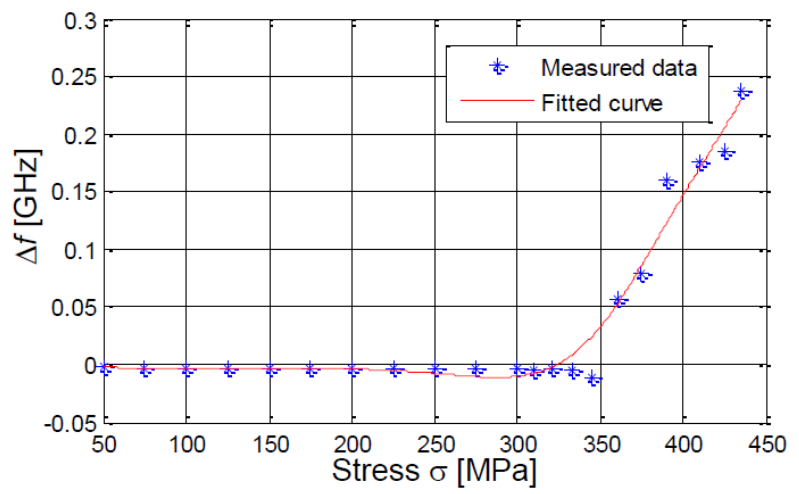

Fig. 8. Results of measurements: a) stress evaluation in elastic region of stress-strain curve (sensor fixed from both sides of patch), b) plastic deformation evaluation (sensor fixed only from one side of patch)

Behavior of proposed sensor was evaluated in two configurations:

1. microstrip antenna is fixed using adhesive from both sides of the patch,

2. microstrip antenna is fixed using adhesive only from one side of the patch.

In case of first configuration, the adhesive connection enables transmission of sample deformation to microstrip sensor. The stress introduce the elongation of sample and sensor, therefore, shifts the antenna resonant frequency. Increasing the load causes the extension of patch antenna, thus the resonant frequency $f_{\mathrm{r}}$ of antenna is decreasing as well as its deviation defined as:

$$
\Delta f=f_{\mathrm{r}, \mathrm{MMPa}}-f_{\mathrm{r}, \mathrm{OMPa}}
$$

where: $f_{r, x M P a}$ - resonant frequency at specified stress $x \mathrm{MPa}, f_{r, 0 \mathrm{MPa}}$ - resonant frequency for sample without external stress.

The results of experiment are presented in the Fig. 8a. One can observe, $\Delta f$ changes linearly with stress in elastic region of stress-strain curve.

In case of second configuration, sensor is not deformed by strain of the sample. Adhesive fixing is utilized only in order to keep the sensor in vicinity of the material. This enables monitoring of materials surface (because of very small penetration depth of high frequency currents) and detection of:

- $\quad$ surface porosity growth (causing change of surface electrical conductivity),

- local narrowing of material just before crack formation,

- $\quad$ crack formation and growth,

- total destruction of the sample.

In case of this configuration results are shown in Fig. 8b. Up to plasticity point the frequency deviation $\Delta f$ is constant. After reaching $\sigma=350 \mathrm{MPa}, \Delta f$ is rapidly increasing. The frequency deviation changes from 0 to $250 \mathrm{MHz}$ in case of totally broken sample. The average sensitivity is $25 \mathrm{MHz} / \mathrm{MPa}$.

\subsection{Damage estimation caused by fluctuating loading conditions}

First analysis of the thermograms sequences obtained for samples after cyclic loading has shown, that due to rapid changes of the temperature during heating phase, and much slower cooling phase, both phases can produce different results. The first step of analysis was removing the heating nonuniformity by using the median 2D filter for each image in the analysed sequence. The median filter mask was big enough to ensure removing the temperature background, but not influence the flaws detection. The result of this operation for exemplary thermogram is shown in Fig. 9. The visible strips are the sample's defects which were produced by the EM sensor during the inspection. These defects are obviously not our in our area of interest. 

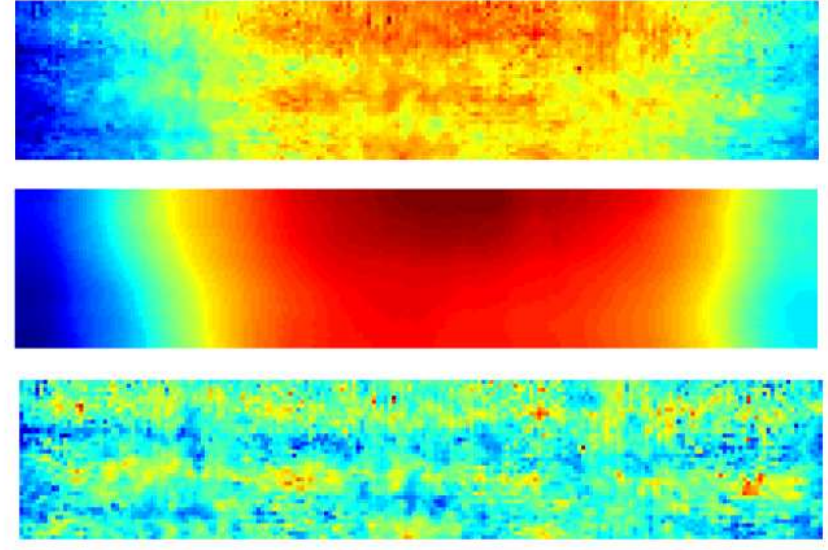

a)

b)

c)

Fig. 9 The process of heating nonuniformity removal. a) exemplary image before the operation, b) the result of median filtration of the exemplary image, c) the result of subtraction a) - b)

In this measurement regime the IR technique can be used only to preliminary determination whether the test sample was subjected to cyclic loading and rough location of fatigue damage. Understanding the process of the material degradation is crucial to carry out the proper results analysis. For the heating phase the image created by computing the mean and for the cooling phase the standard deviation of the temperature value in time is presented in the Fig. 10. As it can be seen the images are noisy, and the information is thus much distorted. The highest values obtained at the samples' very edges should be omitted, as they occur as a result of natural temperature nonuniformity, that arises in those areas. For the images presenting the sample with fatigue damage, one can observe clearly larger values of parameters near the border of the sample. This result indicate the fatigue damage, which is higher in border areas.

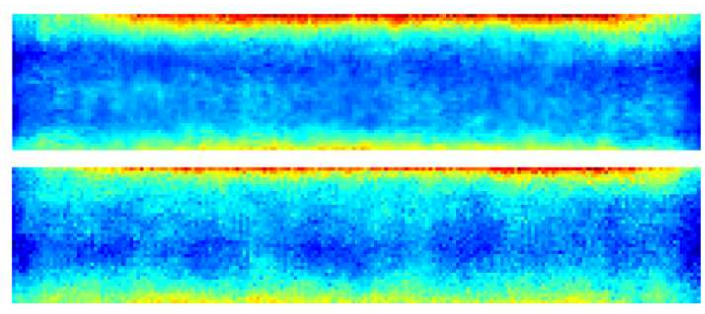

a)
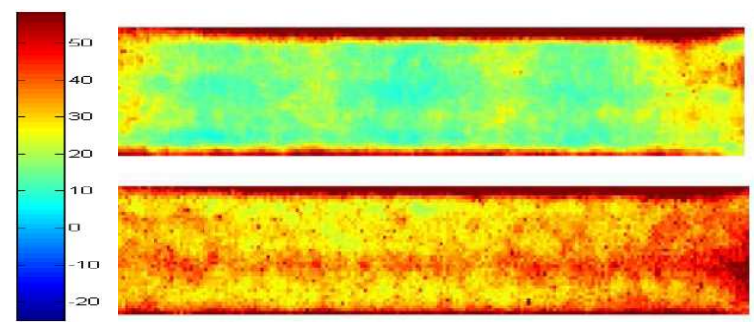

b)

Fig. 10 The results of IRT inspection of the sample. a) mean value obtained for the sequence of heating - upper image sample before fatigue process, bottom - sample after $5,4 \cdot 10^{5}$ fatigue cycles, b) standard deviation value obtained for the sequence of cooling - upper image - sample before fatigue process, bottom - sample after $5,4 \cdot 10^{5}$ fatigue cycles,

To verify the IRT results, the EM testing was conducted. This technique of NDT can be classified as local, and thus should be proceed by previously shown global one, to rough estimation of region of interest.

The chaotic nature of Barkhausen noise signal requires the use of complex algorithms for data analysis. In order to determine the quantitative representation of the Barkhausen noise signal parameters must be calculated. In this article the signals energy is defined as:

$B N_{E N}=\int_{-\infty}^{\infty} u^{2} d t$

where $u$ stands for voltage of measured signal.

Application of $\mathrm{AC}$ magnetization method in non-destructive testing involves monitoring changes of the parameters of the hysteresis minor loop $B(H)$ [15]. Observation of the basic parameters of the loop such as coercivity, remanence or magnetic permeability for maximum or saturation operating conditions creates possibility to detect changes of a material [16]. The observed changes of the loop depend on the state of material damage in area being 


\subsection{1/qirt.2016.039}

tested. Fig. 11 shows the results of deformation monitoring before (Fig. 11 a) and after (Fig. 11 b) carrying out the dynamic loading achieved for EM methods. Each method allows to monitor different aspect of the samples structure changes. The $B N_{\mathrm{EN}}$ distribution allows to observe the changes of stress concentration over the region of interest, while local plastic deformation of the material among others is noticeable by the reduction of the hysteresis loop slope angle to the axis of the field strength $H$. In the area were the deformation bands of greatest damage occurs depicted by the locally rapidly changing value of maximum permeability $\mu_{\max }$ simultaneously low level of $B N_{\mathrm{EN}}$ can be observed. On the other hand, the formation of high concentration of stress area preludes introduction of deformation bands what can be observed by high value $B N_{E N}$ in that area.
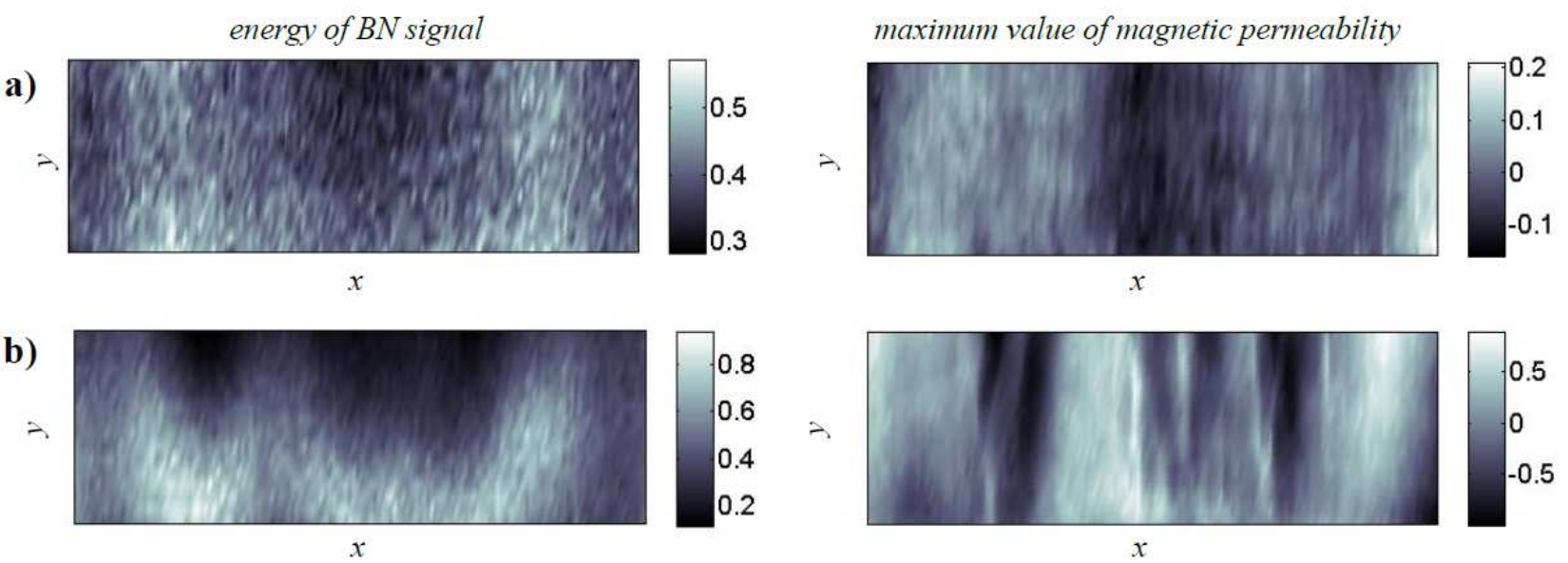

Fig. 11 Results of EM inspection carried out for sample before fatigue process (a) and after $5,4 \cdot 10^{5}$ fatigue cycles; left column - the energy of Barkhausen noise signal, right column - the maximum value of magnetic permeability.

\section{Conclusions}

In this paper the novel approach of multimodal steel constructions state assessment combining information acquired by three complementary inspection techniques was presented. It is obvious, that multi-source approach using methods basing on monitoring changes of physical properties of different type or under different conditions gives the possibility of receiving comprehensive information about the state of evaluated material. The aim of our ongoing research is to evolve inspection multimodal methodology that allows conduction of steel structure degradation and fatigue stage periodic estimation under practical terms. In the real life conditions, the structures are subjected to load which is a combination of static and fluctuating forces, thus we decided to investigate the two processes. In order to assess the impact of each loading conditions the experiments were carried out separately.

In an experiment with static load, thermography was supported by a microwave method. The achieved results allowed to obtain the relationships between selected parameters of both methods (i.e. standard deviation of the temperature and antenna resonant frequency) and the stress value. These results showed a good agreement but still should be understood as preliminary.

The important part of the experiments was dedicated to testing the samples subjected to cyclic loads. The key aspect here is the practical approach, assuming that the test sample is examined after the loading using fatigue machine (not during the process). The aim is to detect defects on the sample after some amount of loading cycles, therefore we were looking for changes in the microstructure of the steel. We were able to pre-determine the suitability of the method of active thermography to a global assessment of the structure in order to determine whether the material was subjected to load and roughly estimate the location of the damage. Using local electromagnetic methods (Barkhausen Noise and AC magnetization) allowed for quantitative estimation of the sample's damage. Also in this case, we are planning further work, mainly related to the testing of samples for various load values in order to explore the full possibilities of the method of active thermography in this aspect.

\section{REFERENCES}

[1 ] Palit Sagar S., et al., "Magnetic Barkhausen emission to evaluate fatigue damage in low carbon structural steel", International Journal of Fatigue, vol. 27, 2005, pp. 317-322

[2 ] Dobmann G., Ciclov D. C., Kurz J. H., NDT and fracture mechanics. How can we improve failure assessment by NDT? Where we are - where we go?, Insight, Vol. 53, No. 12, 2011

[3 ] B. Szymanik, G. Psuj, " Infrared Inspection of Steel Structures Under Load”, QIRT 2015 


\subsection{1/qirt.2016.039}

[4] Maladegue X., Theory and practice of infrared technology for nondestructive testing. JohnWiley and Sons, 2001.

[5] Bzymek, A. Czupryński, M. Fidali, W. Jamrozik and A. Timofiejczuk, Analysis of images recorded during welding processes, 9 International Conference on Quantitative InfraRed Thermography, 2008, Poland

[6 ] Akira DEMIZ, Yukihiro ITO, Hiroshi MATSUDA, Chihiro MORITA, S. Paul Sumitro, Development of defect detection method of steel structures by digital image correlation method using induction heating, International Symposium Non-Destructive Testing in Civil Engineering (NDT-CE) September 15 - 17, 2015, Germany

[7] Psuj G., Chady T., Enokizono M., Observation of material degradation under fatigue and static loading condition using selected electromagnetic NDT methods, Material Science Forum, 721, 120, 2012, 120-126

[8 ] Erdmann J., Huang H., Microwave Antenna Sensors for Fatigue Crack Monitoring Under Lap-joints, ASME 2010 Conference on Smart Materials, Adaptive Structures and Intelligent Systems, Volume 2

[9] Mohammad I., Huang H., Monitoring fatigue crack growth and opening using antenna sensors, Smart Materials and Structures, No. 19, 2010

[10 ] Chocianowicz F., Microstrip antenna based sensor for the detection and monitoring of crack growth in conductive material, Master Thesis, West Pomeranian University of Technology, Szczecin, Poland, 2013

[11 ] Wang W., Ge H., Liu T., Liu M., Study of Patch Antennas for Strain Mesurement, Electromagnetic Nondestructive Evalution (XVIII), 2015

[12 ] Daliri A., Galehdar A., John S., Rowe W. S. T., Ghorbani K.: Slotted Circular Microstrip Patch Antenna Application in Strain Based Structural Health Monitoring, AIAC14 Fourteenth Australian International Aerospace Congress, 2011

[13 ] Balanis C.A.: Antenna theory analysis and design, $3^{\text {rd }}$ ed, US: John Wiley \& Sons Ltd., 2005

[14 ] Lopato P., Psuj G., Herbko M., Maciusowicz M.: Evaluation of stress in steel structures using electromagnetic methods based on monitoring of AC magnetization process and utilization of microstrip antenna sensor, WD 2016 Conference, Lublin

[15 ] Stupakov O., Tomas I.: "Hysteresis minor loop analysis of plastically deformated low-carbon steel", NDT\&E International, 2006, 39, 554-561

[16 ] C. C. H. Lo. et al.: "Structural Characterization, and Magnetic Properties of Steels Subjected To Fatigue", Review In Progress of QNDE 19, 509 (2000), 1597-1604 